\title{
Lean production et modèles de valeur
}

Une approche régulationniste par le travail

Lean production and value models. A regulationist approach through work

\section{Christian du Tertre}

\section{(2) OpenEdition}

Journals

Édition électronique

URL : http://journals.openedition.org/activites/462

DOI : 10.4000 /activites.462

ISSN : 1765-2723

Éditeur

ARPACT - Association Recherches et Pratiques sur les ACTivités

Référence électronique

Christian du Tertre, «Lean production et modèles de valeur », Activités [En ligne], 9-2 | Octobre 2012, mis en ligne le 15 octobre 2012, consulté le 01 mai 2019. URL : http://journals.openedition.org/ activites/462 ; DOI : 10.4000/activites.462

\section{cc) (†) $\ominus$}

Activités est mis à disposition selon les termes de la licence Creative Commons Attribution - Pas d'Utilisation Commerciale - Pas de Modification 4.0 International. 


\title{
Lean production et modèles de valeur Une approche régulationniste par le travail
}

\author{
Christian du Tertre \\ Université Paris Diderot - Paris 7, Ladyss - CNRS, ATEMIS - c.dutertre@atemis-lir.com
}

\begin{abstract}
Lean production and value models. A regulationist approach through work. Lean appeared in an historical and local context (Japan) which has little to do with contemporary economic dynamics. In order to be able to analyze its current impact on work, it would appear necessary to consider new forms of value production and the tensions which occur between the different levels of performance
\end{abstract}

\section{KEYWORDS}

Lean, work, performance, productivity, quality, profitability, regulation

Les organisations connaissent des évolutions régulières. Dans certaines situations, elles subissent de véritables chocs changeant les repères et plus profondément les formes de coopération dans le travail avec de multiples conséquences sur le sens du travail, la santé des salariés, la qualité des services rendus...

L'introduction de méthodes issues de l'expérience japonaise, notamment chez Toyota, généralement dénommées Lean production, dans des entreprises industrielles, mais aussi, aujourd'hui, dans des entreprises de service, dans des organisations publiques ou parapubliques, comme les hôpitaux, pose de nombreuses interrogations quant au devenir du travail.

Cet article resitue le Lean dans le contexte historique de son émergence au Japon, analyse son lien avec les changements de mode de régulation de la performance et présente les contradictions que provoque cette approche de la production face aux évolutions du travail associées aux transformations structurelles de la création de valeur.

\section{1.- Le Lean à son origine : quelques éléments de repères historiques}

L'expression «Lean» apparaît à la fin des années quatre-vingt lorsque des chercheurs du MIT (Massachusetts Institute of Technology) tentent, aux États-Unis, de qualifier les méthodes de gestion de production en vigueur chez Toyota. Mais ces méthodes ont été mises au point et engagées depuis plusieurs années, voire depuis plusieurs décennies dans l'entreprise. Elles relèvent d'une approche spécifique de la qualité totale développée, dès les années soixante, par Toyota sur la base des analyses et des recommandations de T. Ohno (Coriat, 1992).

Cette approche de la «qualité totale » dénommée parfois approche japonaise de la qualité se distingue de l'approche américaine. Aux États-Unis, l'expression «qualité totale » désignait le fait d'appliquer à toute l'entreprise, à tous ses services, les méthodes de "gestion de la qualité ». Il s'agissait donc d'étendre les méthodes de gestion de la qualité qui avaient été 
mises en place dans les ateliers de production au cours des années quarante, cinquante, soixante aux services fonctionnels. Au japon, et plus particulièrement chez Toyota, la notion de «qualité totale » recouvrait l'idée que l'approche de la qualité menée à son terme devait être à même de remettre en cause le découpage fonctionnel des rapports entre services, des rapports entre ateliers, des rapports entre services fonctionnels et ateliers de production (du Tertre, 1990). Certains observateurs parlaient d'une approche relevant d'une pyramide à l'envers vis-à-vis de celle en vogue aux États-Unis qui se limitait à appliquer partout la gestion de la qualité. Pour que les produits réalisés dans un atelier de production progressent en qualité, Toyota envisageait de faire évoluer les relations entre services, entre ateliers, et pas seulement faire évoluer l'organisation du service ou de l'atelier considéré.

Cette approche de la « qualité totale japonaise » supposait que les informations concernant la non-qualité remontent des ateliers vers la direction de l'entreprise pour que soient identifiés et analysés, sur la base d'une approche globale, d'un côté les obstacles à la qualité, de l'autre des leviers d'amélioration. Si l'information de la non-qualité restait cantonnée à l'atelier ou au service dans lesquels la non-qualité était repérée, la capacité d'action était limitée. C'est de cette stratégie que remonte l'idée d'arrêter et de signaler toute anomalie : non pas de la signaler simplement au niveau de l'atelier, mais de la faire remonter. Pour ce faire, il fallait provoquer un incident, arrêter la production. C'est le principe dit «Jidoka» : faire remonter l'information et s'appuyer sur les retours d'expérience pour être à même de faire évoluer les processus; pas seulement le processus interne à l'atelier, mais le processus dans son ensemble. L'événement que provoque l'arrêt de la production oblige à ne pas cantonner l'information sur un plan local.

Cette approche de la qualité va s'adosser à une conception originale, à l'époque, des déterminants de productivité. Au lieu de s'adosser à une logique d'économie d'échelle et à une intensification du travail direct par introduction de nouvelles technologies de production propre à l'industrie de masse en vigueur aux États-Unis et en Europe, Toyota va se préoccuper de créer une industrie «flexible» dont les efforts de rationalisation se concentreront sur l'usage du « capital circulant », c'est-à-dire des « encours de production », des stocks finaux et intermédiaires. La chasse aux gaspillages devient un levier stratégique dans une situation où Toyota était contraint d'impulser une production flexible. En effet, l'industrie automobile japonaise était récente et prenait appui sur un marché intérieur plus étroit que celui des États-Unis ou ceux de l'Europe. De plus, les firmes américaines plus anciennes avaient développé entre les deux guerres une puissante industrie exportatrice, présente au Japon. Rattraper les échelles de production en vigueur aux États-Unis relevait d'une mission impossible. Se construire dans les niches et sur les marchés spéciaux de plus petite taille devenait stratégique. Il fallait, alors dès les années soixante, avant l'apparition du traitement numérique de l'information ${ }^{1}$, impulser une industrie flexible qui exige pour être rentable une attention particulière à la gestion des «encours». C'est le principe du «Juste à temps » (flux continu, flux tiré, zéro stock...).

En définitive, le Lean est apparu au Japon et plus particulièrement chez Toyota sur la base de deux principes :

— un principe de gestion, être informé centralement des éléments de non-qualité repérés localement ;

— un principe économique, accroître la productivité du capital circulant par la flexibilité de l'organisation et la mobilisation subjective des ouvriers : être à même de prendre la décision d'arrêter la chaîne, faire connaître ses idées d'amélioration du processus productif sur la base de son expérience.

1 La « puce » ne sera inventée qu'en 1973 ; son introduction dans la productique au début des années quatrevingts (cf. du Tertre \& Santilli, 1992), 
In fine, au cœur des préoccupations de l'entreprise apparaissent les éléments d'une doctrine industrielle et opératoire originale qui concentre l'attention sur :

— la gestion des flux de matière et leur lisibilité ;

- la généralisation d'indicateurs mesurables ayant un sens tant pour les opérationnels en atelier que pour les concepteurs de processus à la direction de l'entreprise ;

— la circulation régulière de l'information de manière descendante et surtout ascendante, fondée sur l'expérience ;

— l'importance du management de proximité ;

— la mise à plat des processus ;

— une approche systémique et globale se traduisant localement par le renforcement de la standardisation des opérations et des processus.

Cette orientation s'opère dans un cadre macro-économique national d'ensemble au Japon où l'entreprise est conduite à rechercher un modèle productif flexible lui permettant de stabiliser la croissance de niches, dans un marché mondial encore dominé par la production de masse de type fordiste. Ce modèle original est fondé sur des gains de productivité relevant de la flexibilité de l'organisation et de la progression de la qualité des processus.

Dans le fordisme classique, les efforts sont portés sur les investissements technologiques, les équipements étant de plus en plus spécialisés dans une situation où le changement de modèle de voiture conduisait à changer les lignes de production. Le poids grandissant des investissements était amorti par la croissance des volumes de production et de vente. Du côté du travail, la spécialisation des équipements provoquait celle du travail et la répétitivité des tâches. Dans le cas du Japon, la production destinée à des niches reposait sur la flexibilité organisationnelle (les équipements étant faiblement spécialisés - maintien de machinesoutils dites universelles), associée à la mobilisation des salariés. Des équipes d'ouvriers relativement peu spécialisés (vis-à-vis du fordisme classique) réalisaient un travail qui faisait appel à l'intelligence de situation et à l'initiative productive.

\section{2.- Lean, modèles de valeur et modes de régulation de la performance}

Mais la dynamique industrielle qui advient à la fin des années quatre-vingt va être marquée par un changement de mode de régulation de la performance, aux États-Unis, en Europe et au Japon. La prise en considération de ce changement de mode de régulation de la performance est essentielle à saisir, car les effets du Lean, avant et après, ne sont pas les mêmes. Si le Lean peut présenter des aspects positifs vis-à-vis des salariés en situation d'exécution, notamment en atelier de production, avant les années quatre-vingt, la dynamique du Lean va se retourner en son contraire compte tenu d'une évolution majeure concernant les conditions dans lesquelles la valeur est créée.

\section{1.- Le modèle industriel fordien de la valeur}

Dans le modèle industriel de la valeur (du Tertre, 2007), les ressources mobilisées sont essentiellement matérielles. La production engage la transformation de matières premières, de produits semi-finis, tout en mobilisant des équipements, c'est-à-dire des machines-outils plus ou moins automatisées (du Tertre, \& Santilli, 1997, op cité). L'approche de la qualité va s'opérer grâce à des indicateurs relevant de mesures associées à des normes adossées à des étalons de mesure. Le contrôle de la qualité repose ainsi sur la possibilité de vérifier les cotes essentielles des produits semi-finis ou des produits finis à partir de «carottages » en cours et en fin de production.

Les « configurations productives de série » sont au cœur des entreprises industrielles dans la mesure où c'est là que les déterminants industriels de la productivité font leurs effets les plus 
importants : économies d'échelle, intensification du travail, intégration du progrès technique. C'est dans les ateliers de production que repose l'essentiel des gains de productivité. Les services supports ou administratifs sont peu engagés dans des logiques relevant de la recherche de gains de productivité. C'est pourquoi ce sont les ouvriers de production, et en leur sein les ouvriers spécialisés (OS), qui supportent l'essentiel des efforts de productivité d'une entreprise.

La vision opérationnelle de la création de la valeur passe par le caractère séquentiel et parfois simultané des processus de production. Mécaniser les relations entre séquences pour les fluidifier et diminuer les stocks intermédiaires, passe par la conception de chaînes de montage qui, avec l'extension du fordisme, vont être au cœur de la représentation fonctionnelle de la création de la valeur. Cette vision sera, d'ailleurs, reprise en gestion, la fin des années soixante-dix, par M. Porter (du Tertre \& Marielle, 2010).à travers l'expression « chaîne de valeur ». La chaîne de montage exige la standardisation poussée des produits, des produits intermédiaires et des processus. Standardisation, mécanisation et industrialisation vont, ainsi être intimement associées dans les « formes de conscience » tant des responsables d'entreprise que des syndicalistes ou des responsables institutionnels.

Dans une telle approche de la production, le mode de régulation de la performance repose sur trois registres : la qualité, la productivité, la rentabilité. C'est trois registres sont liés les uns aux autres suivant un ordre précis :

- la qualité vient en premier. C'est sur la base d'une stabilisation de la qualité que l'engagement à grande échelle de la production peut être engagé ;

— la productivité vient en second. Une fois cette qualité acquise, l'entreprise cherche à obtenir des gains de productivité en s'appuyant sur les principaux déterminants industriels de la productivité (économies d'échelle, intensification du travail direct, intégration du progrès technique qui favorise l'intégration et les cadences des différentes séquences productives) ;

— la rentabilité vient en troisième. Les gains de productivité permettent de diminuer les coûts unitaires des produits et leurs prix relatifs ce qui va stabiliser et accroître la rentabilité du capital. C'est sur la base des gains de productivité que la rentabilité est assurée. Elle apparaît comme un résultat de la dynamique productive de l'entreprise.

Il faut remarquer que ce mode de régulation de la performance et le modèle de valeur faisant référence sont fondés sur l'extension des marchés internes des différents pays de l'OCDE et la croissance de la demande associée d'une part à la progression de la solvabilité des ménages et, d'autre part, à la généralisation d'un mode de vie centré sur l'acquisition de biens d'équipement. Les effets externes de cette approche de la dynamique productive, notamment, sur l'environnement, ne sont pas pris en compte. Les externalités sont considérées comme hors champ de la performance.

Ce modèle de valeur et ce mode de régulation de la performance sont adossés à un ensemble de dispositifs institutionnels qui garantissent leur pérennité. Ces dispositifs renvoient aux règles et conventions qui structurent le rapport salarial et à celles qui encadrent la concurrence oligopolistique (Boyer, 1986). C'est notamment le cas des conventions concernant le partage des gains de productivité ou les règles de la concurrence qui stabilisent la place de la qualité dans les logiques productives et marchandes. La dynamique de la sphère financière a été conditionnée par le compromis salarial fordiste et par l'encadrement de la concurrence.

\section{2.- Le Lean, un modèle original vis-à-vis du fordisme}

Dans cette situation d'ensemble qui dominait au sein des pays de l'OCDE, le Lean et plus précisément, le mode japonais d'approche des dynamiques industrielles est apparu comme original. Cette approche était très liée à l'environnement macro-économique des entreprises 
de ce pays les poussant à s'engager, bien avant l'Europe et les États-Unis dans le développement de productions en petites séries autorisant la réalisation de produits variés de qualité.

Pour assumer ce projet, l'organisation de la production devait être flexible. Or, dans les années soixante, soixante-dix et même au début des années quatre-vingt, la robotique n'existait pas encore. La flexibilité ne pouvait pas reposer sur l'automatisation flexible, c'est-à-dire sur des technologies industrielles flexibles. La flexibilité comme ressort essentiel des gains de productivité devait, alors, reposer sur l'organisation et un mode particulier de mobilisation de la main-d'œuvre d'exécution.

Un rapport salarial spécifique va être instauré. La flexibilité organisationnelle va être promue octroyant aux ouvriers des espaces d'initiatives (arrêts de la production, innovations de process) et d'échanges (retours d'expériences) que le fordisme classique repoussait. Un lien original va être instauré entre la qualité et la productivité. L'approche de la qualité va être centrée sur les produits intermédiaires de telle manière qu'il était demandé par la hiérarchie de faire «bien du premier coup » même si le temps de réalisation était plus long que les temps standards les plus brefs. Cette approche renouvelée du rapport entre qualité et productivité va être soutenue par des méthodes favorisant la remontée de l'information reposant sur une forme de mobilisation subjective des salariés. Cette dernière était soutenue par la promotion de «l'emploi à vie » et le suivi d'un management de proximité dont la mission était de faire remonter l'expérience.

Ces méthodes constituaient, ainsi, un ensemble de référents éloignés du fordisme classique en tant que mode de régulation de la performance industrielle, même si elles étaient insérées dans une approche de la valeur pouvant être considérée, à première vue, comme assez proche de celle en vigueur dans les autres pays industriels : un, la qualité ; deux, la productivité ; trois, la rentabilité. Ce sont les articulations entre qualité et productivité qui n'étaient pas les mêmes. Faire plus lentement, mais bien du premier coup et éviter ainsi les rebuts ce qui permettait de réduire le capital circulant mobilisé (Japon); faire vite et identifier les rebuts en cours de production pour les écarter progressivement de la ligne de production.

\section{3.- Les années quatre-vingt-dix et l'émergence du mode financiarisé de régulation de la performance}

Les années quatre-vingt et quatre-vingt-dix vont tout bouleverser. Depuis le début des années quatre-vingt, les marchés de biens d'équipement des ménages sont devenus saturés dans les pays de l'OCDE. La grande majorité des ménages était équipée. La demande de ces derniers évoluait vers le renouvellement des biens et le multi-équipement, ces deux démarches de consommation étant très sensibles à l'innovation et à la spécification de la qualité. Les tailles des séries se réduisent, la notion de niches ayant tendance à se généraliser.

La saturation des marchés a provoqué l'intensification de la concurrence et la nécessité pour les entreprises, d'un côté, de fabriquer des produits variés et d'engager de très lourds investissements, notamment en robotique, afin de faire évoluer leurs chaînes de production vers la flexibilité technologique, de l'autre, de trouver de nouveaux débouchés, notamment, dans les pays de l'Est européen ou dans les pays émergents. Cette évolution de la stratégie des entreprises les a conduits à rechercher de nouvelles ressources financières. Les grands groupes, dans leur grande majorité, vont resserrer leur activité sur les segments de production leur apparaissant les plus rentables, les aspects financiers jouant un rôle primordial.

Un nouveau mode de régulation de la performance s'instaure que l'on peut qualifier de mode néo-industriel et financiarisé, les objectifs de rentabilité prévalant sur ceux relevant de la productivité et de la qualité. Les rapports entre les trois registres se sont transformés : 1. la rentabilité ; 2. la productivité ; 3 . la qualité. En effet, la saturation des marchés a un second effet qui ne relève pas seulement des formes de la concurrence, mais du rapport entre gestion 
et production. Tant que les marchés croissaient, la rentabilité était fondée sur les économies d'échelle et apparaissait comme le résultat de ces gains de productivité. Avec la saturation des marchés, les effets d'échelle sont très affaiblis, voire négatifs, les gains de productivité ne sont plus assurés, un risque majeur porte sur le résultat financier. Les entreprises s'engagent alors dans une dynamique gestionnaire ou les aspects financiers influencent directement l'organisation de la production et l'approche même de la productivité comme de la qualité.

La productivité n'est plus abordée de manière physique (tant de pièces ou de produits en tant d'heures de travail), mais de manière monétaire (tant de valeur ajoutée traitée par tant d'heures de travail ou d'hommes). Le passage de l'approche physique de la productivité qui correspond à ce que vit et perçoit l'opérateur au travail, à l'approche en valeur monétaire de la productivité, ce que perçoivent le gestionnaire et le financier, déconnecte l'opérateur du sens du travail dans la mesure où cette grandeur ne dépend plus centralement du travail, mais des évolutions des prix. Cette évolution de l'approche de la productivité a été rendue possible par la généralisation de la notion de «client interne » entre les unités d'une même entreprise et de la notion de «prix interne» ou «prix de session» constamment comparés aux prix de marché. Cette évolution va aussi être facilitée par le passage aux NTIC: d'un côté, la recherche de flexibilité sera adossée à la généralisation de la robotique et plus à la flexibilité de l'organisation et à la mobilisation subjective du travail, de l'autre, l'information circulera par l'intermédiaire de l'informatique qui favorisera le contrôle individuel du travail et son intensification.

La gestion de la qualité va être aussi influencée par l'inversion des ordres de priorités des registres de la performance et le primat du registre de la rentabilité. Vis-à-vis de la clientèle et de l'action commerciale, la qualité est présentée comme centrale, notamment, en termes de produit fini, d'image..., mais comme principe de gestion de la production, la qualité est mise au second plan vis-à-vis de la productivité, au troisième plan vis-à-vis des coûts et de la rentabilité. Les efforts sont portés sur les «entrants coûteux » en dehors de l'appréciation réelle que l'opérateur a de la qualité de son travail, de l'impact de telle ou telle opération sur le devenir final du produit, l'accent étant mis sur les produits intermédiaires et leur vente « aux clients internes».

Dans un tel contexte, le Lean va jouer un rôle très différent dans les organisations productives que celui qu'il avait chez Toyota dans la période historique précédente.

\section{4.- Les effets pervers du Lean associé au mode de régulation financiarisé de la performance}

Les changements de la dynamique économique d'ensemble conduisent à modifier la portée de la diffusion du Lean dans les activités productives contemporaines. La flexibilité va devenir une exigence pour toutes les entreprises, mais dans des conditions très différentes des années soixante. À partir du milieu des années quatre-vingt, cette flexibilité peut être assurée par des robots, la main d'œuvre et l'organisation du travail pouvant passer au second plan. Dans ce nouvel espace de régulation, le Lean va avoir une signification très différente. Son usage va se retourner contre les salariés en situation d'exécution et d'encadrement de proximité. En réalité, la domination de la régulation financiarisée a transformé le contenu social et économique du Lean. Plusieurs séries de remarques méritent d'être formulées.

Les espaces d'autonomie ont changé de finalité et de logique. Ils étaient associés, dans la période précédente, à la décision d'arrêter la ligne pour cause de non-qualité et pour favoriser les retours d'information vers la direction de l'entreprise en vue de faire évoluer les interfaces entre les services et les ateliers de production. En étant adossés à des objectifs de productivité en volume, ils sont, à l'heure actuelle, instrumentalisés vers l'intensification du travail sur un plan local. Cette nouvelle fonction se retourne d'autant plus contre les ouvriers, renommés opérateurs, que les taux de croissance de l'activité se sont ralentis, les gains de 
productivité ayant tendance à provoquer des réductions d'emplois qui remettent en cause «l'emploi à vie». Dans ce nouveau contexte, les opérateurs sont confrontés à des injonctions paradoxales: prendre sur soi et être à l'initiative de la progression de la productivité et prendre le risque d'être à l'origine des pertes d'emplois.

La chasse aux gaspillages a tendance à provoquer des arbitrages défavorables à la qualité au regard des nouvelles tensions qui apparaissent suite au changement de priorité entre qualité et productivité, propre à la financiarisation des entreprises. Cette dynamique engage les opérateurs dans des conflits de conscience entre le respect des consignes et le «travail mal fait (le «sale boulot»).

L'importance accordée par le Lean à la gestion par les flux, favorise la généralisation de la notion de «chaîne de valeur». Or, cette approche de la valeur est le fer de lance des processus de recentrage des entreprises sur leur «cœur de métier» qui se traduisent par l'externalisation des segments les moins rentables, des pertes d'emplois et des changements de convention collective de référence. Le primat de l'approche gestionnaire par les flux et les «prix internes » conduit à mettre en concurrence les sites de production comme les équipes, et à casser toute forme de coopération. Le Lean contribue, ainsi, au délitement des collectifs de travail.

La recherche de «solutions immédiates » propre au Lean favorise la prééminence du court terme sur le moyen et le long terme que l'on constate dans le mode de régulation financiarisé. Cela contribue à enfermer la recherche de réponse aux enjeux productifs dans le périmètre de l'atelier ce qui exclut la prise en compte des externalités, en matière d'environnement, de santé physiologique et mentale, d'inégalités sociales. Dans ce cadre restreint d'action, l'introduction du Lean a tendance à réduire les «investissements immatériels » (du Tertre, 2008) notamment ceux concernant les ressources humaines.

En définitive, le Lean apparaît comme un levier favorisant la domination du mode de régulation financiarisé de la performance des entreprises. Le primat du registre financier sur ceux de la productivité et de la qualité y trouve des justifications d'ordre opérationnel. Le Lean provenant du Japon et plus particulièrement de Toyota les légitime. Le Lean s'appuyant sur des «experts », des gestionnaires «sachant», il est l'occasion d'alliances renouvelées entre les acteurs de la finance et ceux en charge du caractère opérationnel des organisations au sein des entreprises, «managers » ou « consultants » labélisés « Lean ».

\section{3.- Changements structurels, économie servicielle et travail}

Si le Lean peut être analysé comme un levier favorisant la domination du mode financiarisé de régulation de la performance des entreprises, le Lean apparaît, également, comme un dispositif de gestion de la production entrant en contradiction avec les évolutions du travail réel associées aux transformations structurelles des activités productives de ces trente dernières années. Dans ce cadre d'analyse, le Lean apparaît comme un dispositif destructeur de valeur servicelle, au sens où il tend à casser les logiques de coopérations transverses (coopérations entre prestataires et bénéficiaires) propres aux activités de service et à dévoyer la spécificité de l'usage des ressources immatérielles, essentielles dans le travail des salariés en situation d'exécution comme en situation d'encadrement. Il contribue, ainsi, à renforcer les dispositifs empêchant la prise en compte du travail réel.

\section{1.- Passage d'une économie industrielle à une économie servicielle}

La place des activités de service est devenue prépondérante dans la dynamique des économies contemporaines. Cela peut se constater à partir d'analyses quantitatives en termes d'emplois ou de valeur ajoutée. À titre d'illustration, les secteurs industriels ont vu leurs emplois diminuer en France de 4,537 millions à 3,554 millions entre 1990 et 2005, soit une baisse de $21,7 \%$ en 15 ans ; les secteurs de services ont vu leurs emplois croître de 15,038 
millions à 18,301 millions, soit une augmentation de 21,7\%, dans la même période. Au sein des entreprises industrielles, mêmes, les emplois de fabrication ont fortement diminué vis-àvis des emplois de service au point que certains économistes considèrent qu'il n'y a plus de différence à introduire entre industrie et service. Enfin, la structure des investissements a tendance à se déformer; les dépenses relevant des investissements en matériels d'équipement occupant une place de moins en moins grande vis-à-vis des dépenses en « investissements immatériels », c'est-à-dire des dépenses en formation, en recherchedéveloppement, en information et communication, en conseil qui relèvent toutes du financement d'activités de service.

Les services occupent une place prépondérante et jouent un rôle moteur dans la dynamique actuelle. Si au dix-neuvième siècle et au vingtième siècle la croissance était fondée sur des gains de productivité relevant principalement des activités de fabrication, depuis le début des années quatre-vingt-dix et la généralisation des NTIC au sein des activités de service, ces dernières constituent les principaux domaines où les gains de productivité sont générés. Enfin, chacun peut constater que la demande de services a tendance à tirer les activités industrielles en tant qu'activités manufacturières, les produits industriels devenant les équipements mobilisés dans les activités de service. À titre d'exemple, les entreprises de communication sont prêtes à offrir un téléphone portable, si la personne se fidélise aux services offerts.

Cette mutation a des effets considérables sur le travail dans la mesure où dans les activités de service se déploie une dynamique de coproduction entre prestataires et bénéficiaires. Le travail ne se limite pas aux coopérations entre pairs au sein d'un collectif et à la coopération verticale au sein de la ligne hiérarchique, mais s'étend à une coopération transverse c'est-àdire à une coopération engageant les salariés de l'organisation productive et les bénéficiaires du service. De la qualité de cette coopération, de la qualité de la relation qui va s'instaurer entre ces deux types d'acteurs, vont dépendre la qualité du service et l'importance des gains (ou des pertes) de productivité.

Des éléments essentiels de la performance émergent ne relevant plus, uniquement, du périmètre strict de l'entreprise. Le service effectif, toujours distinct du service prescrit, va dépendre, d'une part, de coopérations avec les bénéficiaires qui échappent, pour partie, aux contraintes de subordination des salariés vis-à-vis de leur employeur, d'autre part de contraintes d'accessibilité propres à l'environnement du bénéficiaire. Ce qui pouvait être perçu comme externe à l'entreprise industrielle et à sa performance devient l'une des clés de la performance de la nouvelle organisation industrielle en tant qu'organisation servicielle. L'environnement pénètre le processus de production via l'activité du bénéficiaire que ce bénéficiaire soit un client interne ou externe à l'entreprise.

Ces contraintes d'accessibilité peuvent être identifiées comme relever de cinq domaines :

— des contraintes de synchronisation temporelle; la dynamique servicielle exigeant que s'articule le temps de travail des prestataires avec le temps des bénéficiaires ;

— des contraintes de proximité géographique ou génomique. Le bénéficiaire est souvent amené à se déplacer vers le prestataire (ou inversement, le prestataire vers le bénéficiaire). Ces déplacements prennent du temps et engagent des dépenses ;

- des contraintes culturelles relevant des formes de représentation de la promesse que présente une offre de service, des compétences mobilisées par le bénéficiaire lors de la prestation ;

— des contraintes techniques, le bénéficiaire doit, souvent, être lui même équipé (en matériel informatique, notamment) et disposer des compétences permettant de coopérer ;

— des contraintes juridiques et sociales qui organisent les droits à l'accès sous conditions.

L'environnement de l'activité de coproduction a, ainsi, un impact décisif sur la performance du service, les externalités (les contraintes ou opportunités ne se limitant pas au périmètre 
strict de l'entreprise) jouant un rôle déterminant.

L'approche de la performance et de la valeur servicielle par le Lean est au regard de ce cadre d'analyse contreproductif. La focalisation sur les processus, sur l'aspect séquentiel des flux va à l'encontre du travail réel, à l'encontre des conditions de réalisation des services effectifs. Les principes de rationalisation de l'activité induits par le Lean ne prenant pas en compte les conditions de la coproduction et de son environnement conduisent, actuellement, à détruire de la valeur.

\section{2.- Dimension immatérielle de la valeur et travail}

La seconde transformation structurelle de la dynamique économique tient à l'importance prise par les ressources immatérielles. Si pendant deux siècles, il pouvait être justifié que l'économie se préoccupe essentiellement du mode de mobilisation, d'allocation et d'utilisation des ressources matérielles, ce n'est plus le cas aujourd'hui. Les dépenses effectuées par les organisations pour mobiliser des ressources immatérielles, au sens des ressources non mesurables et non dénombrables comme les compétences, la confiance, la pertinence (d'une organisation), les connaissances, sont devenues considérables. Certes, elles ont toujours existé, mais les sciences économiques -et derrière elle la gestion- pouvaient se justifier de ne pas mettre l'immatériel au cœur de leur approche tant les ressources matérielles, dans l'agriculture comme dans l'industrie, occupaient une place centrale. Aujourd'hui c'est différent. L'aspect stratégique de ces ressources est reconnu par la plupart des observateurs ; la compétitivité hors coût dépendant largement de considérations relevant de l'immatériel, les investissements également.

Or, les ressources immatérielles présentent des caractéristiques distinctes de celles relevant des ressources matérielles. La production en transformant les matières premières comme les produits semi-finis en produits finis transmet la valeur des inputs dans les outputs. Une fois les marchandises réalisées, les ressources matérielles qui ont été utilisées pour les fabriquer, n'existent plus comme ressources. Parallèlement, les outils et les équipements matériels utilisés lors de la production s'usent à hauteur de leur taux d'utilisation. Ils perdent de leur valeur.

Les ressources immatérielles présentent la spécificité suivante : l'expérience que représente la production peut dans certains cas conduire au développement de la ressource. Ce n'est pas mécanique, mais un usage des ressources humaines qui tient compte de la dimension subjective du travail conduit, généralement, à son développement. Les compétences des salariés peuvent à l'occasion de l'expérience productive se développer. La confiance qui s'établit au sein des collectifs de travail comme entre prestataires et bénéficiaires peut à l'occasion de sa mise à l'épreuve lors de la production se renforcer. La pertinence d'un mode opératoire, d'une organisation, de connaissances peut, elle aussi, s'améliorer à l'occasion de l'expérience productive. Ce processus d'accumulation de valeur immatérielle n'est pas mécanique, l'expérience productive pouvant épuiser les compétences et derrière l'employabilité des salariés, détruire la confiance, casser la santé des salariés, mettre à mal la pertinence d'une organisation... Ce potentiel de développement va dépendre de deux séries d'enjeux :

— de l'attention apportée à la qualité de la mise en œuvre du travail de coopération dans ces trois dimensions (horizontale entre pairs, verticale au sein de la ligne hiérarchique, transverse avec le bénéficiaire) lors du processus productif ;

— de la capacité à déployer des procédures permettant de revenir sur l'expérience, en engageant des dynamiques de réflexivité, à propos des trois formes de coopération.

L'approche de l'immatériel ne peut donc pas s'opérer sur la base des principes mis en œuvre dans des logiques gestionnaires à même de ne considérer que ce qui est mesurable. Le Lean représente, de ce point de vue, un ensemble de méthodes en porte à faux vis-à-vis de l'immatériel. Pire la non prise en considération du potentiel de développement de 
l'immatériel conduit à sa dépréciation. À titre d'exemple, la non-prise en compte de l'évolution des compétences des salariés liée à leur expérience conduit à leur démotivation, à leur perte de confiance en eux à l'affaiblissement de cette ressource immatérielle.

\section{3.- Complexité, information et connaissance}

L'incertitude des situations de travail et plus largement des situations productives, associée à la complexité de l'environnement, à son instabilité, à l'importance prise par les activités de services, conduit les organisations à mobiliser et à traiter de plus en plus de données, d'informations et de connaissances. Le temps accordé par chaque salarié, quelles que soient sa compétence et sa responsabilité, au traitement des données, des informations et des connaissances, s'est considérablement accru. L'activité de communication est devenue l'une des dimensions essentielles du travail.

Dans ce cadre, l'usage des NTIC s'est intensifié posant de nouveaux enjeux de régulation aux organisations. Certains concernent le rapport entre le temps de travail et temps hors travail, les NTIC aggravant la porosité des ces deux temporalités, d'autres les capacités de discernement de la pertinence d'une information ou d'une connaissance, d'autres, encore le renouvellement des lieux de délibération face à l'émiettement des collectifs de travail.

La valeur créée par les organisations est très liée à la possibilité de penser ces tensions sur la base de l'expérience, d'élaborer des doctrines en phase avec ces mutations. Il s'agit d'éviter de transposer des notions qui avaient encore une certaine pertinence dans les périodes dominées par les activités industrielles, sur les situations de travail contemporaines. L'application de procédures dites industrielles dans le domaine de la communication conduit à des pertes de temps considérables, des incompréhensions sources de tensions contreproductives. L'importation du Lean dans ces configurations de travail représente une régression considérable. L'activité déontique au sein des collectifs disparaissant dans les approches procédurales.

\section{Conclusion : Modèle de valeur et perspective de développement durable}

La diffusion des méthodes d'organisation et de gestion de la production se référant au Lean est symptomatique d'une part de la domination des modes de régulation de la performance par la finance, d'autre part, des difficultés à concevoir et à soutenir l'émergence d'un modèle de valeur qui tienne compte des évolutions du travail réel, et qui puisse faire référence. Les acteurs économiques, sociaux et institutionnels en lien avec le monde du travail, les chercheurs en sciences sociales et les consultants adoptant une posture de recherche sont interpelés dans leur capacité à coopérer afin que puisse se constituer un autre rapport à la valeur, un rapport à la valeur qui tienne compte du travail réel des salariés.

La mise en perspective du travail dans le cadre du développement durable peut être une orientation. En effet, le devenir du travail est très directement lié à un mode de développement qui soit en capacité d'associer les préoccupations écologiques, sociales et économiques tout en envisageant de nouvelles formes de gouvernance, notamment, des organisations. Les considérations servicielles de l'approche de la valeur constituent des cheminements utiles pour progresser dans cette voie.

Il s'agit, alors d'opposer à l'introduction du Lean trois types d'initiatives représentant trois types d'investissements immatériels en lien avec le travail et le développement des capacités humaines :

— des procédures d'évaluation (Hubault, \& du Tertre, 2008) fondées sur les retours d'expérience concernant les formes de la coopération dans le travail. Il s'agit de considérer la différence entre le prescrit et le réel non pas comme un dysfonctionnement à 
réduire, voire supprimer mais comme un écart à identifier et à valoriser. Ces procédures d'évaluation s'opposent aux procédures dites d'évaluation quantitative relevant essentiellement des résultats ;

- des dispositifs de professionnalisation s'appuyant sur les retours d'expérience et l'avancée des connaissances, notamment dans les domaines des sciences sociales et des sciences humaines ;

— des dispositifs d'innovation fondés sur l'expérience de travail et prenant en compte les exigences de développement durable.

La promotion de ces trois types de dispositifs institutionnels serait une façon de faire face à la diffusion du Lean et des méfaits du mode industriel et financiarisé de régulation de la performance des organisations tant privées que publiques ou parapubliques.

\section{BIBLIOGRAPHIE}

Boyer, R. (1986). La théorie de la régulation, une analyse critique. Paris: La Découverte.

Coriat, B. (1992) Penser à l'envers. Paris: Éditions Christian Bourgois.

Hubault, F., \& Tertre du, C. (2008). Le travail d'évaluation. In F. Hubault (Ed.), Évaluation du travail, travail d'évaluation. Actes du colloque des 4-6 juin 2007 (pp. 95-114). Toulouse: Éditions Octarès.

Tertre du, C. (1990). La qualité dans le bâtiment : une analyse critique. Cahiers du PUCA, Ministère de l'équipement et du logement.

Tertre du, C. (2007). Economie de la fonctionnalité, performance et Développement Durable. In E. Heurgon (Ed.), Economie des services et Développement Durable (pp. 39-49). Paris: L'Harmattan, Paris.

Tertre du, C. (2008). Investissements immatériels et patrimoine collectif immatériel. In C. Laurent \& C. du Tertre (Eds.), Secteurs et territoire dans les régulations émergentes (pp. 73-90). Paris: Edition l'Harmattan.

Tertre du, C., \& Marielle, B. (2010). De la valeur industrielle à la valeur servicielle : les conséquences sur le travail et l'action syndicale. Rapport ATEMIS pour la confédération de la CFDT ; www.atemis-lir.com

Tertre du, C., \& Santilli, G. (1992). Automatisation et travail. Paris: PUF, collection économie en liberté.

\section{RESUME}

Le Lean est apparu dans une configuration historique et locale (le Japon) qui n'a plus grand-chose à voir avec la dynamique économique contemporaine. Pour être à même d'analyser son impact actuel sur le travail, il apparaît nécessaire de prendre en compte les nouvelles formes de production de valeur et les tensions qui apparaissent entre les différents registres de la performance.

\section{MOTS CLES}

Lean, travail, performance, productivité, qualité, rentabilité, régulation

\section{REFERENCEMENT}

Tertre du, C. (2012). Lean production et modèle de valeur. Une approche régulationniste par le travail. Activités, 9(2), 168-178, http://www.activites.org/v9n.pdf

Article soumis le 22 mars 2012, accepté pour publication le 4 septembre 2012 